\title{
Trade-off Between Energy and Area Spectral Efficiencies of Cell Zooming and BSs Cooperation
}

\author{
Mansour M. Aldosari and Khairi Ashour Hamdi \\ School of Electrical and Electronic Engineering, \\ The University of Manchester, Manchester M16 1QD, U.K. \\ Email: mansour.aldosari-2@postgrad.manchester.ac.uk, k.hamdi@manchester.ac.uk.
}

\begin{abstract}
Reducing power consumption in wireless communication networks has been the concern of many researchers over the last decade. In this paper we study the performance of two recently proposed methods for increasing the energy efficiency of green cellular systems, namely base station (BSs) cooperation and cell zooming techniques. We investigate the trade-off between the area spectral efficiency (ASE) and energy efficiency (EE) of these two models and compare this with the basic cellular system, which is used as a reference. We derive novel mathematical expressions for the ergodic capacity in these three systems, which are then are used to compute the ASE and EE of the different scenarios.
\end{abstract}

Index terms - Base station cooperation, cell zooming, area spectral efficiency, energy efficiency.

\section{INTRODUCTION}

In Italy, for example, communication operators use about $1 \%$ of the total power consumed nationally [1], which means that communication networks use more than $2 T W h$ of power per year-a figure which is expected to increase tenfold in the next ten years. Annual subscriber energy consumption is about $49 \mathrm{KWh} /$ year, and the number of sites in 2007 was about 3.3 million, which is expected to increase to more than 11 million by 2020 [2]. Increasing energy demands and costs, supported by the keen desire of telecommunication networks to save energy, encourage engineers to design new techniques to improve network energy efficiency. Similarly, cellular communication network manufacturers researchers need to improve their products' energy efficiency, which might be achieved via improvements to hardware and software relating to network components. Power consumed by secondgeneration (2G) BSs (GSM) and third-generation (3G) BSs (UMTS) has reduced to $800 \mathrm{~W}$ and $430 \mathrm{~W}$, respectively [3]. Equipment capabilities should support the many and varied ideas and techniques applied by planning engineers, whilst effort should focus on accessing network segments which consume about 60 to $80 \%$ [1] of the total power used by the whole communication network. BS sites consume $80 \%$ of the power used by cellular network operators [2]; however, reducing the number of active BSs in one area will help us to reach our objective, although this reduction should be carried out according to specific criteria and rules. Customer behaviour and traffic intensity are the main factors that will help us to decide how we shall apply these rules, so cells which have lower data traffic compared to other cells should therefore be turned to sleep mode [4] - active cells will cover the areas that were originally covered by these sleeping cells (passive BSs), which in turn will increase the output power of the active cells' base stations. Users in the sleep cells might notice a slight difference in the quality of service (QoS) before and after switching their base station to sleep mode. In this situation, the UE will have to increase its output power, due to the increased distance between it and the active BS, which might ultimately reduce battery lifetime. In this paper, we apply a deep-sleep mode, because BSs at zero load consume $80 \%$ to $90 \%$ of power at full load [5]. The main purpose of our proposal is to increase energy efficiency and by default cut $\mathrm{CO}_{2}$ emissions in wireless communication networks. $\mathrm{CO}_{2}$ emitted by communication networks was measured in 2007 at $0.2 \%$ of global emissions, a figure which is expected to double by 2020 [6].

Traffic streams in cellular communication networks vary during the day, depending on many factors such as human activities and user habits. However, during under-utilisation periods, BSs in sleep mode would be a suitable way of increasing energy efficiency. In this case, frequency management is considered a good option for mitigating inter-cell interference caused by the zooming out of active BSs, due to decreases in the number of UEs during low traffic periods.

Recent relevant research (e.g. [7]) has not considered cochannel interference but has instead investigated EE performance and the influence of SE on EE in MIMO systems over Rayleigh fading channels. [8], for instance, did not investigate the trade-off between ASE and EE for different models, e.g. cell zooming and cooperative BSs. [9] did investigate the energy-spectral efficiency trade-off in virtual MIMO cellular systems, but the author did not consider SIR or compare different models.

The main contributions of this paper are as follows:

- Derives novel equations to calculate the ergodic capacity for different cellular models such as basic, zooming and cooperative models.

- Calculates and compares the energy efficiency and area spectral efficiency of these models.

- Calculates and compares the trade-off between energy and area spectral efficiencies for these models.

- Verifies the above results using a Monte Carlo simulation.

\section{ANAlysis OF THE BASIC MODEL}

In this section, we consider a basic cellular system with four cells in each cluster. The basic model has two situations- 


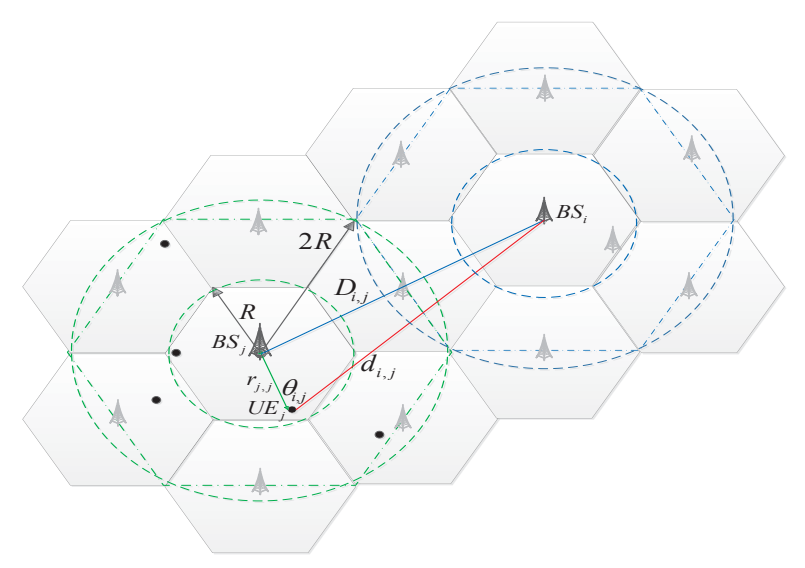

Figure 1. Cellular system with four cells in each cluster. The bold BSs are in active mode. Larger circles show the zooming boundaries.

the first is the full traffic mode, while the second situation is the partial/low traffic mode, where the number of active UEs will be reduced by $75 \%$ during any network underutilisation period. In both situations, we calculate the spectral and energy efficiencies of the basic model. The performance of this basic system will then be used as a norm against which to study possible improvements offered as a result of using base station cooperation or cell zooming. Without any loss of generality, consider a reference-user equipment (UE)to be arbitrary UE in an arbitrary cell (which will call the home cell), Fig. 1. In the basic model, we assume the home cell is active and therefore the useful signal is received from the home base station. The signal-to-interference plus noise (SINR) experienced by the reference UE takes the form:

$$
\mathrm{SINR}=\frac{P_{0}\left|a_{0}\right|^{2} r^{-\beta}}{\sum_{i=1}^{N} P_{i}\left|a_{i}\right|^{2} d_{i}^{-\beta}(r, \theta)+N_{0}}
$$

where $P_{i}, i=0,1, \ldots, N$ is the transmitted power of the firsttier interfering base stations $\left(\mathrm{BS}_{i}\right)$ and $N=6$ in this case, $\beta$ is the path loss exponent, $(r, \theta)$ are random variables which represent the polar coordinates of the UE relative to its home base station and

$$
d_{i}(r, \theta)=\sqrt{D_{i}^{2}+r^{2}+2 D_{i} r \cos \left(\theta+\phi_{i}\right)}
$$

is the distance from $\mathrm{BS}_{i}$ to the reference UE. Here $\left(D_{i}, \phi_{i}\right)$ are the polar coordinates of $\mathrm{BS}_{i}$ (relative to the home $\mathrm{BS}$ ), which depends on the frequency re-use factor of the cellular system.

In (1), $\left\{\left|a_{0}\right|^{2},\left|a_{1}\right|^{2}, \ldots\left|a_{N}\right|^{2}\right\}$ are the channel gains. In this paper, we assume Rayleigh fading, where $\left\{\left|a_{0}\right|^{2},\left|a_{1}\right|^{2}, \ldots\left|a_{N}\right|^{2}\right\}$ are independent and exponentially distributed random variables.

Therefore, the ergodic (average) capacity achieved by an arbitrary user can be estimated by using the average of the Shannon capacity formula[10]:

$$
C=\mathbb{E}\left[\log _{2}\left(1+\frac{\left|a_{0}\right|^{2} r^{-\beta}}{\sum_{i=1}^{N}\left|a_{i}\right|^{2} d_{i}^{-\beta}(r, \theta)+1 / \mathrm{SNR}}\right)\right]
$$

$[\mathrm{b} / \mathrm{s} / \mathrm{Hz}]$ where $\mathrm{SNR}=\frac{P_{o}}{N_{0}}$ is the signal-t- noise ratio, and we have assume that no power control is used where $P_{0}=P_{1}=\ldots=$ $P_{N}$.

The expectation in (3) is to be applied with respect to the $N+3$ non-negative random variables $\left\{\left|a_{0}\right|^{2},\left|a_{1}\right|^{2}, \ldots\left|a_{N}\right|^{2}, r, \theta\right\}$. Classical methods employed to evaluate such an average require at least $N+3$ fold of numerical integrations, which will make the process very complicated. In order to reduce the computational complexity of (3), we next invoke a non-direct method which simplifies greatly the required computational efficiency.

Lemma 1: Let

$$
\begin{aligned}
& C(r, \theta) \\
& =\mathbb{E}\left[\ln \left(1+\frac{\left|a_{0}\right|^{2} r^{-\beta}}{\sum_{i=1}^{N}\left|a_{i}\right|^{2} d_{i}^{-\beta}(r, \theta)+1 / \mathrm{SNR}}\right) \mid r, \theta\right]
\end{aligned}
$$

where the expectation applies to the $N+1$ independent exponential random variables $\left\{\left|a_{0}\right|^{2},\left|a_{1}\right|^{2}, \ldots\left|a_{N}\right|^{2}\right\}$ which represent the channel gains in Rayleigh fading. Then

$$
\begin{aligned}
C(r, \theta)=\int_{0}^{\infty} \frac{1}{z}\left(1-\frac{1}{1+z r^{-\beta}}\right) \prod_{n=1}^{N} & \frac{1}{1+z d_{n}^{-\beta}(r, \theta)} \\
& \times e^{-\frac{z}{\mathrm{SNR}}} d z .
\end{aligned}
$$

Proof of Lemma 1: The following proof is based on [11, Eq. 6], which states that

$$
\ln (1+t)=\int_{0}^{\infty} \frac{1}{s}\left(1-e^{-s t}\right) e^{-s} d s, \quad t \geq 0 .
$$

Let $t=\frac{\left|a_{0}\right|^{2} r^{-\beta}}{\sum_{i=1}^{N}\left|a_{i}\right|^{2} d_{i}^{-\beta}(r, \theta)+1 / \mathrm{SNR}}$ in (6). Then

$$
\begin{aligned}
& \ln \left(1+\frac{\left|a_{0}\right|^{2} r^{-\beta}}{\sum_{i=1}^{N}\left|a_{i}\right|^{2} d_{i}^{-\beta}(r, \theta)+1 / \mathrm{SNR}}\right) \\
& =\int_{0}^{\infty} \frac{1}{s}\left(1-e^{-s \frac{\left|a_{0}\right|^{2} r^{-\beta}}{\sum_{i=1}^{N}\left|a_{i}\right|^{2} d_{i}^{-\beta}(r, \theta)+1 / \mathrm{SNR}}}\right) e^{-s} d s .
\end{aligned}
$$

Substitute $s=z\left(\sum_{i=1}^{N}\left|a_{i}\right|^{2} d_{i}^{-\beta}(r, \theta)+1 / \mathrm{SNR}\right)$. Then $d s=\left(\sum_{i=1}^{N}\left|a_{i}\right|^{2} d_{i}^{-\beta}(r, \theta)+1 / \mathrm{SNR}\right) d z$ and we obtain from (7)

$$
\begin{gathered}
\ln \left(1+\frac{\left|a_{0}\right|^{2} r^{-\beta}}{\sum_{i=1}^{N}\left|a_{i}\right|^{2} d_{i}^{-\beta}(r, \theta)+1 / \mathrm{SNR}}\right) \\
=\int_{0}^{\infty} \frac{1}{z} e^{-\frac{z}{\mathrm{SNR}}}\left(1-e^{-s\left|a_{0}\right|^{2} r^{-\beta}}\right) \\
\times \prod_{i=1}^{N} e^{-s\left|a_{i}\right|^{2} d_{i}^{-\beta}(r, \theta)} d s .
\end{gathered}
$$

Now, since the channel gains $\left\{\left|a_{0}\right|^{2},\left|a_{1}\right|^{2}, \ldots\left|a_{6}\right|^{2}\right\}$ are assumed to be independent exponential random variables, we have for the conditional average (conditioned on the polar 
coordinates of the reference user $(r, \theta)$ )

$$
\begin{array}{r}
C(r, \theta) \\
=\mathbb{E}\left[\ln \left(1+\frac{\left|a_{0}\right|^{2} r^{-\beta}}{\sum_{i=1}^{6}\left|a_{i}\right|^{2} d_{i}^{-\beta}(r, \theta)+1 / \mathrm{SNR}}\right) \mid r, \theta\right] \\
=\int_{0}^{\infty} \frac{1}{s} e^{-\frac{s}{\mathrm{SNR}}}\left(1-\mathbb{E}\left[e^{-s\left|a_{0}\right|^{2} r^{-\beta}} \mid r\right]\right) \\
\times \prod_{i=1}^{6} \mathbb{E}\left[e^{-s\left|a_{i}\right|^{2} d_{i}^{-\beta}(r, \theta)} \mid r, \theta\right] d s .
\end{array}
$$

Using (4) and (3) we arrive at the following expression for the ergodic capacity of an arbitrary connection in the basic model:

$$
C=\log _{2}(e) \int_{R_{0}}^{R} \int_{0}^{2 \pi} C(r, \theta) \frac{2 r}{2 \pi\left(R^{2}-R_{0}^{2}\right)} d \theta d r
$$

where $R_{0}$ is the closest distance between the UE and its home base station. We have also used the fact that the probability density function (PDF) of the UEs' polar coordinate $(r, \theta)$ relative to their $\mathrm{BS}$ are given by

$$
f_{r}(r)=\frac{2 r-R_{0}^{2}}{R^{2}-R_{0}^{2}}, \quad R_{0} \leq r \leq R
$$

and

$$
f_{\theta}(\theta)=\frac{1}{2 \pi} \quad 0 \leq \theta \leq 2 \pi .
$$

Area spectral efficiency (ASE) is one of the most important factors inherent in different types of communication networks. Network design engineers aim at increasing ASE, especially as service providers make huge requests regarding spectrum frequency. ASE can be expressed as in [12]:

$$
\mathrm{ASE}=\frac{L C}{A n} \quad[\mathrm{~b} / \mathrm{s} / \mathrm{Hz} / \text { Cluster }]
$$

where $C$ is the ergodic capacity of a single user, given in (10), $A$ is the cell area, $n$ is the number of cells in each cluster and $L$ is the total number of active users.

On the other hand, energy efficiency (EE) can be estimated as follows [13]:

$$
\eta_{E E}=\frac{\epsilon P_{T D}+P_{C}}{W L C} \quad[\mathrm{~J} / \mathrm{bit}] .
$$

where $W$ represents cell bandwidth and $\epsilon P_{T D}+P_{C}$ the total power consumed by a base station. Here, $P_{T D}$ is the transmit power-dependent (which reflects the power consumed by the amplifier and feeder losses) and $P_{C}$ is the circuit power (found in hardware such as air-conditioning systems, backup batteries, signal processors and rectifiers). Note that $P_{C}$ is a transmit power-independent.

Parameter $\epsilon$ in (14) is the scaling factor of load-dependent power consumption [14].

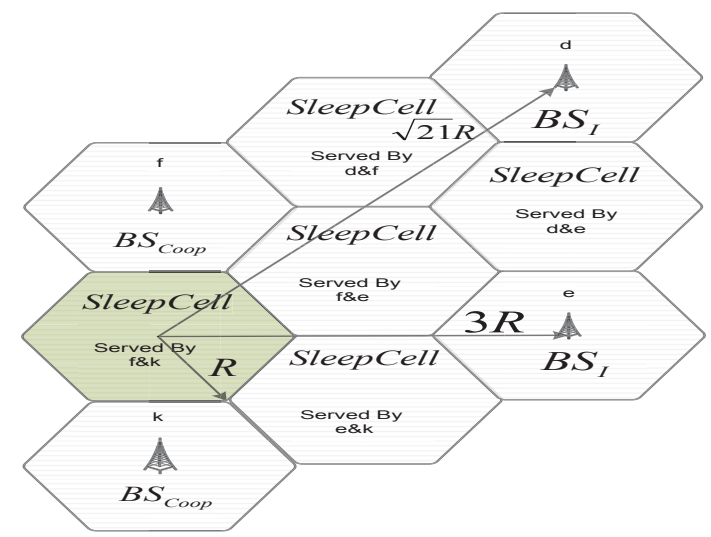

Figure 2. Cooperative model, which shows cooperative BSs in bold; interfering and sleep cells are in between.

\section{The Cooperative Model}

In the cooperative model, we assume that the home base station is in sleep mode and the reference UE is served by two neighbouring base stations $(M=2)$. Fig. 2 shows such an example.

In this case, it can be demonstrated that ergodic capacity takes the form

$$
C=\mathbb{E}\left[\log _{2}\left(1+\frac{\sum_{m=1}^{M}\left|a_{m}\right|^{2} d_{m}^{-\beta}(r, \theta)}{\sum_{i=1}^{N}\left|a_{i}\right|^{2} d_{i}^{-\beta}(r, \theta)+1 / \mathrm{SNR}}\right)\right]
$$

$(r, \theta)$ in this model are random variables which represent the polar coordinates of the UE relative to its home base station, which is in sleep mode, and the two cooperative BSs.

In order to evaluate the expectation in (15) we invoke the following Lemma.

Lemma 2 : Let

$C_{M}(r, \theta)=\mathbb{E}\left[\ln \left(1+\frac{\sum_{m=1}^{2}\left|a_{m}\right|^{2} d_{m}^{-\beta}(r, \theta)}{\sum_{i=1}^{6}\left|a_{i}\right|^{2} d_{i}^{-\beta}(r, \theta)+1 / \mathrm{SNR}}\right) \mid r, \theta\right]_{(16)}$

Then

$$
\begin{aligned}
C_{M}(r, \theta)=\int_{0}^{\infty} \frac{1}{z}\left(1-\prod_{m=1}^{2} \frac{1}{1+z d_{m}^{-\beta}(r, \theta)}\right) \\
\prod_{i=1}^{6} \frac{1}{1+z d_{i}^{-\beta}(r, \theta)} e^{-\frac{z}{\mathrm{SNR}}} d z .
\end{aligned}
$$

Proof of Lemma 2: The proof is similar to Lemma 1.

\section{The Cell Zooming Model}

In this model, we applied the same procedures used for the basic model above, to derive the zooming model capacity equation by considering the increase in the re-use distance. The cell radius is $2 R$, where $R$ is the basic model's cell radius [15], [16]. Fig. 3-B shows a system deployment and interfering $\mathrm{BSs}$, which are illustrated in bold. In this figure we rearranged the frequency spectrum allocation of the system due to the 


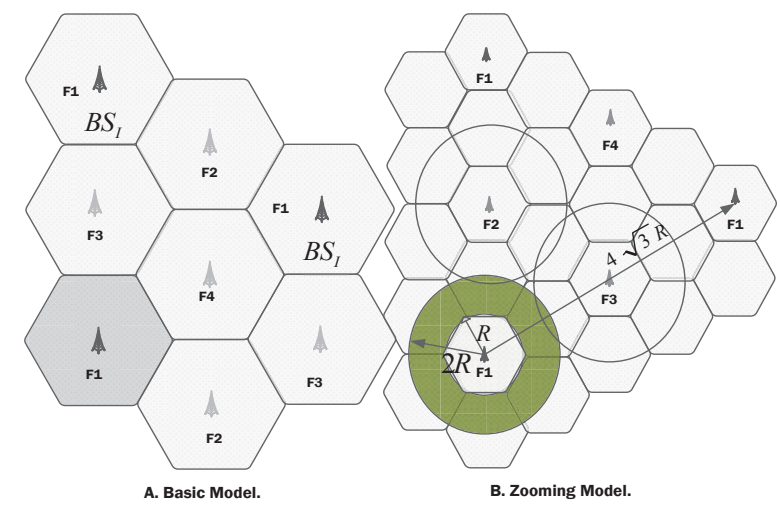

Figure 3. In both models, interfering BSs are shown in bold, while dim BSs are in sleep mode.

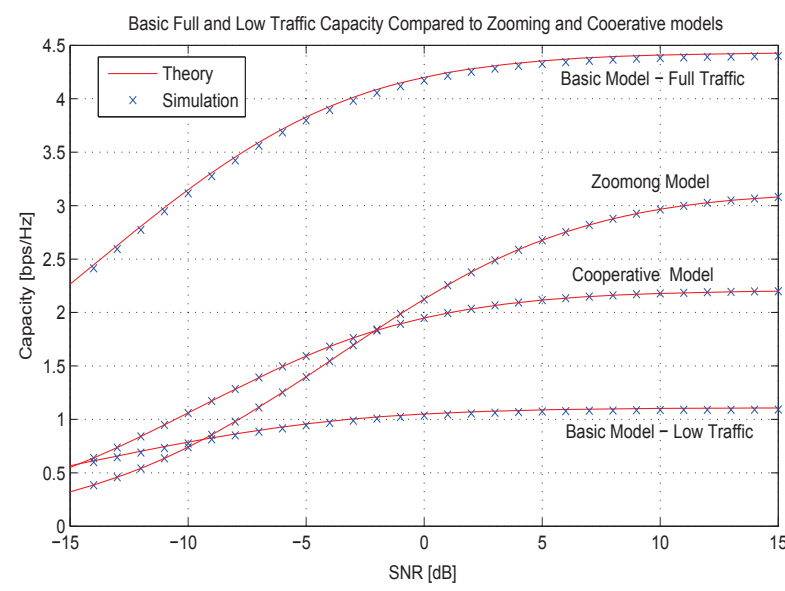

Figure 4. Capacity vs. signal-to-noise ratio for basic, zooming and cooperative models using path loss exponents equal to 3 .

change in the size of the (virtual) cells. According to this configuration, we have six interfering BSs that use the same frequency set. The re-use distance $D_{i}=4 \sqrt{3} R=2 \sqrt{3} R_{z}$, where $R_{z}$ is the cell radius after zooming out, which equals $2 R$. We consider that three cells out of four will be turned to sleep mode, and the remaining active cell will double its covering distance to cover half of the surrounding sleep cells. The other half will be covered by the other active cells etc.[17], [18].

\section{Simulation}

Using MATLAB to simulate the mathematical process above, we achieve the following:

1) User $U E_{j}$ 's position is randomly located by referring to the $B S_{j}$ as follows:

a) Generate $u_{j}$ as a pseudo-random number uniformly distributed in $[1,0]$ as

$$
r_{j, j}=R_{0}+\left(R-R_{0}\right) \sqrt{u_{j}} .
$$

b) Calculate the user's position according to (11). c) Generate channel gain $\left|a_{j, j}\right|^{2}$ of the useful signal between $U E_{j}$ and $B S_{j}$.

2) Calculate distance $d_{i, j}$ of $U E_{j}$, by referring to $B S_{i}$ as follows:

a) Generate the angle between the $U E_{j}$ and $B S_{i}$ randomly by using (19) below, following which add $60^{\circ}$ for the next interfering $B S_{i}$ (we considered the first-tier interfering $B S s)$.

$$
\theta_{i, j}=2 \pi \sqrt{u_{i}}
$$

b) Use equation (2) to calculate the distance $d_{i, j}$ between $U E_{j}$ and $B S_{i}$, where frequency re-use distance $D_{i, j}$ is fixed and is equal to $2 \sqrt{3} R$.

c) Generate the independent channel gain $\left|a_{i, j}\right|^{2}$ of the interfering signal between $U E_{j}$ and the six interfering $B S_{i}$.

3) Use equation (3) to calculate spectral efficiency.

4) Repeat the above process 10,000 times and compare it with the result of the mathematical equation in (9) for the basic model.

5) Apply the same procedures to the cooperative and zooming models.

For the purpose of numerical examples, we assume in this section that in the basic model $N=6$ cochannel cells with coordinates $D_{i}=2 \sqrt{3} R \forall i$ and $\phi_{i}=$ $\left\{30^{\circ}, 90^{\circ}, 150^{\circ}, 210^{\circ}, 270^{\circ}, 330^{\circ}\right\}$.

\section{NUMERICAL RESUlTS}

In this work, we found that the basic model during full traffic periods is better than zooming and cooperative model capacity and ASE, due to using smaller cells in the basic model, while during low traffic periods we found that applying zooming and cooperative models will increase capacity and ASE, as shown in Figs 4 and 5. During low traffic periods, the capacity for the basic model at $15[\mathrm{~dB}]$ is 1.1 , while it is 2.3 and $3.1[\mathrm{~b} / \mathrm{s} / \mathrm{Hz}]$ for the cooperative and zooming models, respectively.

Fig. 5 shows that the value of ASE for the basic models during low traffic periods at $15 \mathrm{~dB}$ is 0.5 , while it is 3.9 and 1.9 [b/s/Hz/Cluster] for the cooperative and zooming models, respectively, which means that there are a massive enhancements in ASE when we use the cooperative and cell zooming models compared to the basic model during low traffic periods. Fig. 6 shows improvements in EE when we apply the cell zooming and cooperative BSs techniques compared to the basic model during low and full traffic periods, due to a decrease in the number of active BSs per cluster. During low traffic periods the $\mathrm{EE}$ of the basic mode degrades severely due to the reduction in the number of users, as shown in Fig. 6. Fig. 6 shows that the EEs of cooperative and zooming models are improved when compared to the basic system at both low and full traffic periods, due to the reduction in the number of active BSs. From the same figure at $2[\mathrm{~b} / \mathrm{s} / \mathrm{Hz}]$, the EE is about 30 $[\mathrm{mJ} / \mathrm{b}]$ for the basic model during a low traffic period, while it is 3 and $12[\mathrm{~mJ} / \mathrm{b}]$ for cooperative and zooming models, respectively. However, the EE degrades for the basic model during low traffic periods and is enhanced when we apply the cooperative and zooming models. The EE improves by about 


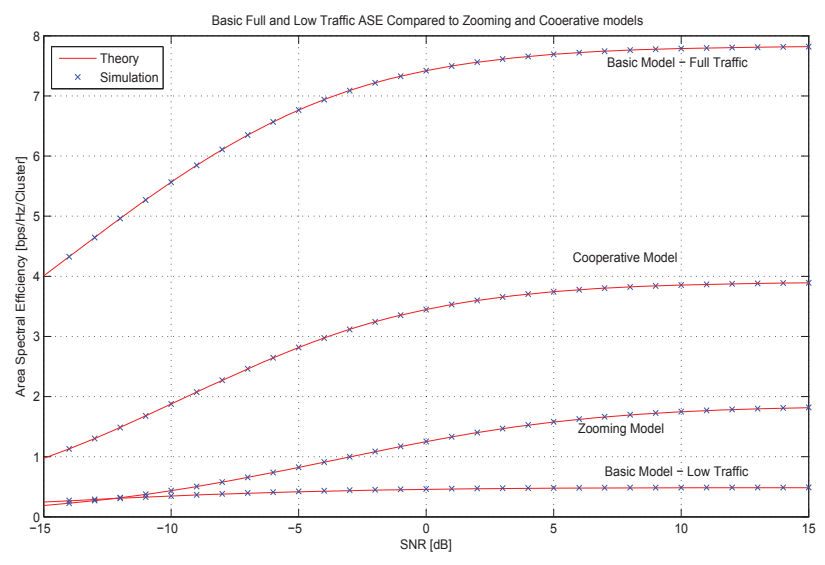

Figure 5. Area spectral efficiency vs. signal-to-noise ratio for basic, zooming and cooperative models using path loss exponents equal to 3 .

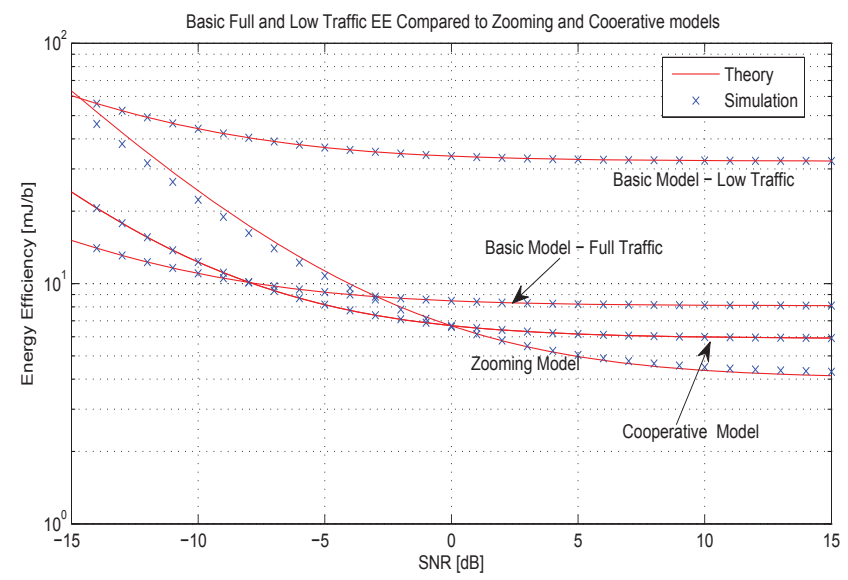

Figure 6. Energy efficiency vs. signal-to-noise ratio for zooming, cooperative and basic models using path loss exponents equal to 3 .

$50 \%$ and $25 \%$ when we apply the cooperative and zooming models compared to the basic model at full load, while it improves by $86 \%$ and $80 \%$ when we apply cooperative and zooming models, respectively, compared to the basic model at partial load. The EE of the zooming model improves after $10[\mathrm{~dB}]$ SNR compared to its cooperative counterpart. Fig. 7shows the trade-off between EE and ASE for all models, which degrades for the basic model during low traffic periods compared to full traffic periods and improves in the zooming and cooperative models. Furthermore, in the zooming and cooperative models we acquire higher ASE with lower power consumption compared to the basic model, as shown in Fig. 7.

\section{SUMMARY AND CONCLUSION}

This paper investigated the trade-off between ASE and EE for different models in dense urban areas, by using small-scale Rayleigh fading and by considering co-channel interference. Cooperative and zooming models were studied and compared with a basic cellular communication model during both full and low traffic periods, in order to find

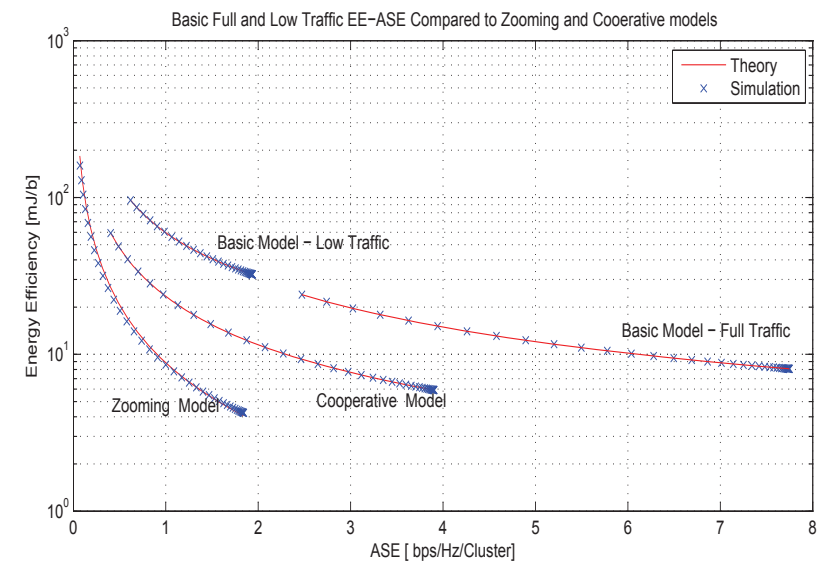

Figure 7. Area spectral efficiency vs energy efficiency for basic, cooperative and zooming models at path loss exponents equal to 3 .

ways to improve power efficiency. Frequency management was considered, especially when we applied cell zooming, to mitigate interference between BSs that use the same frequency set. We derived mathematical equations for the three different models to calculate ASE and EE, and we also verified the results using a Monte-Carlo simulation. The zooming model shows better performance in both ASE and EE compared to the cooperative and basic models, while the cooperative model is better than the basic model in ASE and EE trade-off.

\section{REFERENCES}

[1] M. Marsan, S. Buzzi, D. Ciullo, and M. Meo, "Optimal energy savings in cellular access networks," in Communications Workshops, 2009. ICC Workshops 2009. IEEE International Conference on, 2009, pp. 1-5.

[2] G. Auer, V. Giannini, C. Desset, I. Godor, P. Skillermark, M. Olsson, M. Imran, D. Sabella, M. Gonzalez, O. Blume, and A. Fehske, "How much energy is needed to run a wireless network?" Wireless Communications, IEEE, vol. 18, no. 5, pp. 40-49, 2011.

[3] V. Mancuso and S. Alouf, "Reducing costs and pollution in cellular networks," Communications Magazine, IEEE, vol. 49, no. 8, pp. 63-71, 2011.

[4] I. Ashraf, F. Boccardi, and L. Ho, "Power savings in small cell deployments via sleep mode techniques," in Personal, Indoor and Mobile Radio Communications Workshops (PIMRC Workshops), 2010 IEEE 21st International Symposium on, 2010, pp. 307-311.

[5] M. Marsan, S. Buzzi, D. Ciullo, and M. Meo, "Multiple daily base station switch-offs in cellular networks," in Communications and Electronics (ICCE), 2012 Fourth International Conference on, 2012, pp. 245-250.

[6] A. Fehske, G. Fettweis, J. Malmodin, and G. Biczok, "The global footprint of mobile communications: The ecological and economic perspective," Communications Magazine, IEEE, vol. 49, no. 8, pp. 5562, 2011.

[7] J. Jiang, M. Dianati, M. Imran, R. Tafazolli, and Y. Chen, "On the relation between energy efficiency and spectral efficiency of multipleantenna systems," pp. 1-1, 2013.

[8] P. Yu, Q. Yang, F. Fu, and K. S. Kwak, "Inter-cell cooperation aided dynamic base station switching for energy efficient cellular networks," in Communications (APCC), 2012 18th Asia-Pacific Conference on, 2012, pp. $159-163$.

[9] X. Hong, Y. Jie, C.-X. Wang, J. Shi, and X. Ge, "Energy-spectral efficiency trade-off in virtual mimo cellular systems," Selected Areas in Communications, IEEE Journal on, vol. 31, no. 10, pp. 2128-2140, 2013.

[10] K. Hamdi, "A useful lemma for capacity analysis of fading interference channels," Communications, IEEE Transactions on, vol. 58, no. 2, pp. 411-416, 2010. 
[11] K. A. Hamdi, "Capacity of mrc on correlated rician fading channels," Communications, IEEE Transactions on, vol. 56, no. 5, pp. 708-711, 2008.

[12] M.-S. Alouini and A. Goldsmith, "Area spectral efficiency of cellular mobile radio systems," Vehicular Technology, IEEE Transactions on, vol. 48, no. 4, pp. 1047-1066, 1999

[13] L. Deng, Y. Rui, P. Cheng, J. Zhang, Q. Zhang, and M. Li, "A unified energy efficiency and spectral efficiency tradeoff metric in wireless networks," Communications Letters, IEEE, vol. 17, no. 1, pp. 55-58, 2013.

[14] Y. S. Soh, T. Q. Quek, M. Kountouris, and H. Shin, "Energy efficient heterogeneous cellular networks," Selected Areas in Communications, IEEE Journal on, vol. 31, no. 5, pp. 840-850, 2013.

[15] Z. Niu, Y. Wu, J. Gong, and Z. Yang, "Cell zooming for cost-efficient green cellular networks," Communications Magazine, IEEE, vol. 48, no. 11, pp. 74-79, 2010.

[16] A. Conte, A. Feki, S. Buzzi, D. Ciullo, M. Meo, and M. Ajmone Marsan, "Cell wilting and blossoming for energy efficiency," Wireless Communications, IEEE, vol. 18, no. 5, pp. 50-57, 2011

[17] E. Oh, B. Krishnamachari, X. Liu, and Z. Niu, "Toward dynamic energyefficient operation of cellular network infrastructure," Communications Magazine, IEEE, vol. 49, no. 6, pp. 56-61, 2011.

[18] F. Han, Z. Safar, W. Lin, Y. Chen, and K. Liu, "Energy-efficient cellular network operation via base station cooperation," in Communications (ICC), 2012 IEEE International Conference on, 2012, pp. 4374-4378. 